\title{
SPLINE DIFFERENCE SCHEME FOR TWO-PARAMETER SINGULARLY PERTURBED PARTIAL DIFFERENTIAL EQUATIONS
}

\author{
W. K. ZAHRA*, M. S. EL-AZAB AND ASHRAF M. EL MHLAWY
}

\begin{abstract}
In this paper, we construct a numerical method to solve singularly perturbed one-dimensional parabolic convection-diffusion problems. We use Euler method with uniform step size for temporal discretization and exponential-spline scheme on spatial uniform mesh of Shishkin type for full discretization. We show that the resulting method is uniformly convergent with respect to diffusion parameter. An extensive amount of analysis has been carried out to prove the uniform convergence with respect to the singular perturbation parameter. The obtained numerical results show that the method is efficient, stable and reliable for solving convection-diffusion problem accurately even involving diffusion parameter.
\end{abstract}

AMS Mathematics Subject Classification : 65H05.

Key words and phrases : Singular perturbation, Convection-diffusion problem, Exponential spline,Shishkin mesh, Error estimates, Uniform convergence.

\section{Introduction}

In the present paper, we are concerned with the numerical approximation of the partial differential equation:

$$
u_{t}-\varepsilon_{d} u_{x x}+\varepsilon_{c} p(x) u_{x}+q(x) u=f(x, t), \quad(x, t) \in Q_{T} .
$$

Here $Q_{T}=\Omega \times I, \quad \Omega \equiv(a, b), \quad \partial \Omega \equiv\{a, b\}, \quad I \equiv(0, T)$, and $a$ and $b$ are real positive constants. We consider equation (1) associated with the Dirichlet boundary conditions

$$
u=0,(x, t) \in \partial \Omega \times I
$$

and the initial condition

$$
u(x, 0)=u_{0}(x), \quad x \in \Omega,
$$

Received January 30, 2013. Revised May 9, 2013. Accepted May 27, 2013. ${ }^{*}$ Corresponding author.

(c) 2014 Korean SIGCAM and KSCAM. 
where $u(x, t)$ is the dependent variable, $x$ and $t$ are the independent variables and $f, p, q$ and $u_{0}$ are given functions of their arguments. It is usually assumed that $0<\varepsilon_{c} \ll 1$ and $0<\varepsilon_{d} \ll 1$.

This problem encloses both the reaction-diffusion problem when $\varepsilon_{c}=0$ and the convection-diffusion problem when $\varepsilon_{c}=1$. These problems occur naturally in various fields of science and engineering, such as, heat transfer with large Peclet numbers, combustion, nuclear engineering, control theory, elasticity, fluid mechanics, aerodynamics, quantum mechanics, optimal control, chemicalreactor theory, convection-diffusion process and geophysics; see $[9,10,18]$.

There has been a lot of effort in developing numerical methods for the solution of singular perturbation problems that are uniformly convergent. Clavero et al.[1] and Kadalbajoo et al. [4] gives a uniform convergent numerical method with respect to the diffusion parameter to solve the one-dimensional timedependent convection-diffusion problem. They used the implicit Euler method for the time discretization and the simple upwind finite difference scheme on a Shishkin mesh for the spatial discretization. Ramos [7] presented an exponentially fitted method for singularly perturbed, one-dimensional (convectiondiffusion) parabolic problems, and showed its uniform convergence in the perturbation parameter. Surla and Jerkovic [13] considered a singularly perturbed boundary value problem using a spline collocation method. Sakai and Usmani $[11,12]$ gave a new concept of B-spline in terms of hyperbolic and trigonometric splines which are different from the earlier ones. It is proved that the hyperbolic and trigonometric B-splines are characterized by a convolution of some special exponential functions and a characteristic function on the interval $[0$, 1]. Sharma and Kaushik [14] presented a numerical approximation of a singularly perturbed time delayed convection diffusion problem on a rectangular domain. Finally, Zahra and El Mhlawy [18] developed a numerical method to solve two-parameter singularly perturbed semi-Linear boundary value problems. This method is based on exponential spline with a piecewise uniform Shishkin mesh which is shown to be uniformly convergent independent of mesh parameters and perturbation parameter $\varepsilon_{c}$ and $\varepsilon_{d}$.

Our contribution in this paper is to introduce a full discretization scheme based on Rothe's approximation in time discretization and on the exponential spline approximation in the spatial discretization for solving singularly perturbed, one-dimensional time-dependent convection-diffusion problem associated with homogeneous Dirichlet boundary conditions. It starts with the discretization in time by the 2-point Euler backward finite difference method in the time variable. After that we deal with the exponential spline method for the solution of the time discretized problem to compute the unknown function and the obtained system of algebraic equations is solved by iterative methods. Moreover, we obtain error estimates for the approximation process. The proposed technique is programmed using Matlab \%, and used in solving the proposed problem. 
The rest of the paper is organized as follows: In section 2, we give some notations, assumptions and definitions, In $\S 3$, a time discretization scheme for the continuous problem is proposed in addition to the derivation of some a priori estimates. In $\S 4$, we prove the convergence results and error estimates of the semidiscretization scheme described in $\S 3$. We describe in $\S 5$ a full discretization scheme based on Rothe- exponential spline methods and the error estimates of the full discretized solution are derived. Finally, numerical results and discussions are presented and comparisons are made with other solutions in $\S 6$.

\section{Notations, assumptions and definitions}

In the sequel, we will denote by $(.,$.$) either the standard inner product in$ $L_{2}=L_{2}(\Omega)$ or the pairing between $V \equiv H_{0}^{1}(\Omega)$ and $V^{*} \equiv H^{-1}$ (see e.g. [11]). we use the symbols | . |, $\|$. $\|$ and $\|.\|_{*}$ as the norms in $L_{2}(\Omega), V, V^{*}$, respectively. By $\rightarrow, \stackrel{w}{\longrightarrow}$, we mean the strong and weak convergence. Also, we introduce some notations concerning the time discretization of our problem.

$$
\delta z_{i}=\frac{z_{i}-z_{i-1}}{\tau}, \quad \bar{z}^{i}=\frac{1}{\tau} \int_{I_{i}} z(., t) d t, \quad 1 \leq i \leq n
$$

for any given family $\left\{z_{i}\right\}_{i=0}^{n}$. The following elementary relations will be used in the following analysis:

$$
\sum_{i=1}^{s}\left(u_{i}-u_{i-1}, u_{i}\right)=\left|u_{s}\right|^{2}-\left|u_{0}\right|^{2}+\sum_{i=1}^{s}\left|u_{i}-u_{i-1}\right|^{2},
$$

and Young's inequality

$$
a b \leq \frac{\varepsilon}{2} a^{2}+\frac{1}{2 \varepsilon} b^{2} \quad \forall a, b \in R,
$$

where $\varepsilon$ is a small constant. We will assume, throughout this work, the following hypotheses on the given data.

(H1) The function $f: \quad \Omega \times I \rightarrow R$ is Lipschitz continuous in the sense of

$$
\left|f(x, t)-f\left(x, t^{\prime}\right)\right| \leq c\left|t-t^{\prime}\right|, \quad \forall t, \quad t^{\prime} \in I,
$$

with $f(0,0)=0$.

(H2) The functions $p(x)$ and $q(x)$ are smooth functions and satisfy

$$
p_{l} \leq p(x) \leq p_{u}, q_{l} \leq q(x) \leq q_{u} \forall x \in \Omega,
$$

(H3) $u_{0} \in H_{0}^{1}(\Omega)$

Under these assumptions, we can define the weak solution of problem (1)-(2).

Definition 2.1. The measurable function $u \in C\left(I ; L_{2}(\Omega)\right) \bigcap L_{2}(I ; V)$ with $\partial_{t} u \in L_{2}\left(I ; V^{*}\right)$ and $u(x, 0)=u_{0}(x)$ is said to be a weak (variational) solution of (1)-(3) if and only if the integral identity

$$
\left(u_{t}, \varphi\right)+\varepsilon_{d}\left(u_{x}, \varphi_{x}\right)+\varepsilon_{c}\left(p(x) u_{x}, \varphi\right)+(q(x) u, \varphi)=(f, \varphi),
$$

holds for all $\varphi \in V$ and a.e. $t \in I$. 
Remark. It is evident that for any functions $\alpha, \beta \in H^{1}(\Omega)$ and $\gamma \in L_{2}(\Omega)$

$$
\left|\left(\alpha \beta_{x}, \gamma\right)\right| \leq\|\alpha\|^{1 / 2}|\alpha|^{1 / 2}\left|\beta_{x}\right||\gamma| \leq\|\alpha\|\|\beta\|\|\gamma\|,
$$

\section{The temporal discretization scheme, A prior estimate}

Our main goal is to approximate (1)-(3) from a numerical point of view and to prove its convergence. The scheme starts with the discretization in time by the 2-point Euler backward finite difference method in the time variable, so that the problem is converted into a linear system of differential equations that easily solved numerically at each subsequent time level. Let $m$ be a positive integer and subdivide the time interval $I$ by the points $t_{j}$, where $t_{j}=j \tau, \tau=T / m$, $j=0,1, \cdots, m$. The suggested discretization scheme of problem (8) consists of the following problem (in the weak sense):

Find $w_{j} \cong u\left(., t_{j}\right) \in V, \quad j=1, \cdots, m$ such that $w_{0}=u_{0}$ in $\Omega$,

$$
\left(\delta w_{j}, \varphi\right)+\varepsilon_{d}\left(\partial_{x} w_{j}, \partial_{x} \varphi\right)+\varepsilon_{c}\left(p(x) \partial_{x} w_{j}, \varphi\right)+\left(q(x) w_{j}, \varphi\right)=\left(f_{j}, \varphi\right) \forall \varphi \in V,
$$

where $\partial_{x}$ denotes to the derivative with respect to $x$ and $f_{j}=f\left(x, t_{j}\right)$.

The existence of a weak solution $w_{j} \in V$ is proved in [2]. By means of $w_{j}$, $(j=0,1, \cdots, m)$ determined by the proposed scheme $10-11$ in each time step $t_{j}$, we introduce the following piecewise linear functions (Rothe functions)

$$
w^{m}(0)=u_{0}, w^{m}(t)=w_{j-1}+\left(t-t_{j-1}\right) \delta w_{j}, \text { for } t \in\left(t_{j-1}, t_{j}\right], j=0,1, \ldots m
$$

and the corresponding step function

$$
\bar{w}^{m}(t)= \begin{cases}w_{j} & \text { for } t \in\left(t_{j-1}, t_{j}\right] \\ u_{0} & \text { for } t=0\end{cases}
$$

Using the notation of Rothe function and its corresponding step function, a piecewise constant interpolation of equation 11 over $I$ for each $\varphi \in V$ yields

$$
\left(\partial_{t} w^{m}, \varphi\right)+\varepsilon_{d}\left(\partial_{x} \bar{w}_{\tau}^{m}, \varphi\right)+\varepsilon_{c}\left(p \partial_{x} \bar{w}^{m}, \partial_{x} \varphi\right)+\left(q \bar{w}^{m}, \varphi\right)=\left(\bar{f}^{m}, \varphi\right),
$$

where $t_{j-1} \leq t \leq t_{j}$ and $\bar{f}^{m}=f\left(., \bar{t}^{m}\right)$ with $\bar{t}^{m}=t_{j}$.

In order to show the stability of the discrete solution and prove the convergence results, we shall derive some a priori estimates.

Lemma 3.1. Under the assumptions (H1)-(H3), there exists a positive constant $C$ such that for any $s$,

$$
\sum_{j=1}^{s} \tau\left|\delta w_{j}\right|^{2}+\max _{s}\left\|w_{s}\right\|^{2}+\max _{s}\left|w_{s}\right|^{2}+\sum_{j=1}^{s}\left\|w_{j}-w_{j-1}\right\|^{2}+\sum_{j=1}^{s}\left|w_{j}-w_{j-1}\right|^{2} \leq C .
$$

Proof. Let us choose $\varphi=\tau \delta w_{j}$ in 11 and summing over $j$ from 1 to $s$, we obtain

$$
\begin{aligned}
\sum_{j=1}^{s}\left(\delta w_{j}, \tau \delta w_{j}\right) & +\varepsilon_{d} \sum_{j=1}^{s}\left(\partial_{x} w_{j}, \partial_{x}\left(w_{j}-w_{j-1}\right)\right)+\varepsilon_{c} \sum_{j=1}^{s}\left(p(x) \partial_{x} w_{j}, \tau \delta w_{j}\right) \\
& +\sum_{j=1}^{s}\left(q(x) w_{j}, w_{j}-w_{j-1}\right)=\sum_{j=1}^{s} \tau\left(f_{j}, \delta w_{j}\right)
\end{aligned}
$$


Taking into consideration (4), the second and the fourth terms are estimated by

$$
\begin{aligned}
\varepsilon_{d} \sum_{j=1}^{s}\left(\partial_{x} w_{j}, \partial_{x}\left(w_{j}-w_{j-1}\right)\right) & =\frac{\varepsilon_{d}}{2}\left(\left|\partial_{x} w_{s}\right|^{2}-\left|\partial_{x} u_{0}\right|^{2}+\sum_{j=1}^{s}\left|\partial_{x}\left(w_{j}-w_{j-1}\right)\right|^{2}\right) \\
& =\frac{\varepsilon_{d}}{2}\left(\left\|w_{s}\right\|^{2}-\left\|u_{0}\right\|^{2}+\sum_{j=1}^{s}\left\|w_{j}-w_{j-1}\right\|^{2}\right), \\
\left|\sum_{j=1}^{s}\left(q(x) w_{j}, w_{j}-w_{j-1}\right)\right| & \geq \frac{q_{l}}{2}\left(\left|w_{s}\right|^{2}-\left|u_{0}\right|^{2}+\sum_{j=1}^{s}\left|w_{j}-w_{j-1}\right|^{2}\right)
\end{aligned}
$$

With the aid of 11, Younge's inequality (5) and (9), we estimate the third term by

$$
\left|\varepsilon_{c} \sum_{j=1}^{s}\left(p(x) \partial_{x} w_{j}, \tau \delta w_{j}\right)\right| \leq \frac{\varepsilon_{c} p_{u}}{2 \varepsilon} \sum_{j=1}^{s} \tau\left\|w_{j}\right\|^{2}+2 \varepsilon \varepsilon_{c} \sum_{j=1}^{s} \tau\left|\delta w_{j}\right|^{2}
$$

The last term is estimated by the use of (6) as follows

$$
\left|\sum_{j=1}^{s} \tau\left(f_{j}, \delta w_{j}\right)\right| \leq \sum_{j=1}^{s} \tau\left|f_{j}\right|\left|\delta w_{j}\right| \leq \frac{1}{2 \varepsilon} \sum_{j=1}^{s} \tau\left|f_{j}\right|^{2}+2 \varepsilon \sum_{j=1}^{j} \tau\left|\delta w_{j}\right|^{2}
$$

Collecting (16)-(20), using (H3), and choosing $\varepsilon$ sufficiently small, we get

$$
\begin{aligned}
& \sum_{j=1}^{s} \tau\left|\delta w_{j}\right|^{2}+\frac{\varepsilon_{d}}{2}\left\|w_{s}\right\|^{2}+\frac{\varepsilon_{d}}{2} \sum_{j=1}^{s}\left\|w_{j}-w_{j-1}\right\|^{2} \\
\leq & c_{\varepsilon} \sum_{j=1}^{s} \tau\left|f_{i}\right|^{2}+\varepsilon \sum_{j=1}^{s} \tau\left|\delta w_{j}\right|^{2}+\frac{\varepsilon_{c} p_{u}}{2 \varepsilon} \sum_{j=1}^{s} \tau\left\|w_{j}\right\|^{2}+2 \varepsilon \varepsilon_{c} \sum_{j=1}^{s} \tau\left|\delta w_{j}\right|^{2}
\end{aligned}
$$

Applying Gronwall's inequality, we conclude the proof.

Lemma 3.2. Uniformly with respect to $n$ one has

$$
\begin{gathered}
\left\|\partial_{t} w^{m}\right\|_{L_{2}\left(I ; L_{2}(\Omega)\right)}^{2} \leq C\left\|\bar{w}^{m}\right\|_{C(I ; V)}^{2} \leq C,\left\|w^{m}\right\|_{C(I ; V)}^{2} \leq C \\
\left\|w^{m}-\bar{w}^{m}\right\|_{L_{2}\left(I ; L_{2}(\Omega)\right)}^{2} \leq \frac{C}{m^{2}},\left\|w^{m}-\bar{w}^{m}\right\|_{L_{2}(I ; V)}^{2} \leq \frac{C}{m}
\end{gathered}
$$

Proof. The estimates (22) and (23) are a consequence of (15). By the use of the identity

$$
\left|w^{m}-\bar{w}^{m}\right| \leq 2\left|w_{j}-w_{j-1}\right|,
$$

the estimates $(22)_{3}$, and $(23)$ are a consequence of $(15)_{2},(15)_{3}$ and the definitions of $w^{m}$ and $\bar{w}^{m}$ and thus the proof completes. 


\section{Convergence results}

This section is devoted to proving the convergence of the proposed scheme and estimating its accuracy. Before we are able to prove convergence we need to prove the compactness of $\bar{w}^{m}$ in $L_{2}\left(I ; L_{2}(\Omega)\right)$ which is a consequence of the following assertion.

Lemma 4.1. The estimate

$$
\int_{0}^{T-z}\left|\bar{w}^{m}(t+z)-\bar{w}^{m}(t)\right|^{2} \leq C\left(z+\frac{1}{m}\right),
$$

holds uniformly for $0<z<z_{0}$ and $n$.

Proof. [3] We sum up (12) for $i=s+1, \cdots, s+k$ considering $\varphi=\left(w_{s+k}-w_{s}\right) \tau$. Then we sum it up for $s=1, \cdots, m-k$ and obtain the estimate

$$
\sum_{s=0}^{m-k}\left|u_{s+k}-u_{s}\right|^{2} \tau \leq C k \tau
$$

Hence for $k \tau \leq z \leq(k+1) \tau$ we conclude the desired estimate.

Theorem 4.1. There exists $u \in L_{2}\left(I ; L_{2}(\Omega)\right) \bigcap H^{1}\left(I ; L_{2}(\Omega)\right)$ such that

$$
\left.\begin{array}{lll}
w^{m} \longrightarrow u & & \\
w^{m} \stackrel{w}{\longrightarrow} u, \quad \bar{w}^{m} \stackrel{w}{\longrightarrow} u & \text { in } L_{2}\left(I ; L_{2}(I ; V)\right) \\
\partial_{t} w^{m} \stackrel{w}{\longrightarrow} \partial_{t} u & & \text { in } L_{2}\left(I ; L_{2}(\Omega)\right)
\end{array}\right\}
$$

(in the sense of subsequences). Moreover, we have

$$
\left\|u-\bar{w}^{m}\right\|_{L_{2}\left(I ; L_{2}(\Omega)\right)}^{2}+\left\|u-\bar{w}^{m}\right\|_{L_{2}(I ; V)}^{2} \leq \frac{C}{m^{2}} .
$$

Proof. The estimate $(22)_{2}$ implies

$$
\int_{Q}\left(\bar{w}^{m}(x+y, t)-\bar{w}^{m}(x, t)\right)^{2} \leq C|y|, \forall|y| \leq y_{0}
$$

Hence, from lemma 4.1, $\left\{\bar{w}^{m}\right\}$ is compact in $L_{2}\left(I ; L_{2}(\Omega)\right)$ because of Kolmogorov's compactness argument. So we can conclude that $w^{n} \rightarrow u$ and $\bar{w}^{m} \rightarrow u$ in $L_{2}\left(I ; L_{2}(\Omega)\right)$ and also pointwise in $Q$. Also by the fact that $\bar{w}^{m} \in$ $L_{2}\left(I ; L_{2}(\Omega)\right) \cap C(I ; V)$, we obtain that $\bar{w}^{m}$ is weakly convergent in $L_{2}(I ; V)$; i.e.

$$
\partial_{x} \bar{w}^{m} \stackrel{w}{\longrightarrow} \partial_{x} u i n L_{2}(I ; V)
$$

and this implies that

$$
\left(\partial_{x} \bar{w}_{\tau}^{m}, \varphi\right) \rightarrow\left(u_{x}, \varphi\right), \forall \varphi \in V
$$

It remains to prove that $\partial_{t} w^{m}=\partial_{t} u$. For each $t \in I$, by lemma $3.3, \partial_{t} w^{m}$ is uniformly bounded in the reflexive Banach space $L_{2}\left(I ; L_{2}(\Omega)\right)$ and hence has a subsequence which converges weakly to an element $\xi \in V^{*}$ (Eberlin-Smulian theorem [6]). Thus

$$
\partial_{t} w^{m} \stackrel{w}{\longrightarrow} \xi i n V^{*} \forall t \in I .
$$


Using Fubini theorem, we get

$$
\left(w^{m}(t)-u_{0}, \chi\right)=\int_{0}^{t}\left(\partial_{t} w^{m}, \chi\right) d t
$$

Taking the limit as $n \rightarrow \infty$, we obtain

$$
\left(u-u_{0}, \chi\right)=\int_{0}^{t}(\xi, \chi) d t
$$

which implies

$$
\left(u-u_{0}-\int_{0}^{t} \xi(t) d t, \chi\right)=0 .
$$

Therefore, we have $\partial_{t} u=\xi$. Using the above discussions and the fact that and hence $\bar{f}^{n} \rightarrow f$ in $L_{2}\left(I ; L_{2}(\Omega)\right)$ and passing with $n \rightarrow \infty$ in (14) the proof is complete.

To obtain an error estimate, let us start by introducing the following additional notation $e_{u}=u(t)-\bar{w}^{m}$. We now take the difference between (8) and (14) we easily obtain the equality for every $\varphi \in V$

$$
\begin{aligned}
& \left(\partial_{t}\left(u-w^{m}\right), \varphi\right)+\varepsilon_{d}\left(\nabla\left(u-\bar{w}^{m}\right), \nabla \varphi\right)+\varepsilon_{c}\left(p\left(\partial_{x} u-\bar{w}^{m}\right), \varphi\right)+\left(q\left(u-\bar{w}^{m}\right), \varphi\right) \\
= & \left(\left(f-\bar{f}^{m}\right), \varphi\right) .
\end{aligned}
$$

Take $\varphi=e_{u}$ and write the new equation as $I+I I+I I I+I V=V$. To begin with, we split the parabolic term into two parts $I=I_{1}+I_{2}$, where

$$
\begin{gathered}
I_{1}=\frac{1}{2} \frac{d}{d t}\left|e_{u}\right|^{2} \\
\left|I_{2}\right| \leq\left|w^{m}-\bar{w}^{m}\right|\left|e_{u}\right| \leq 2 \xi\left|w^{m}-\bar{w}^{m}\right|^{2}+\frac{1}{2 \xi}\left|e_{u}\right|^{2}
\end{gathered}
$$

The second term of equation (37) is bounded by

$$
|I I| \leq\left|\varepsilon_{d}\left(\nabla\left(u-\bar{w}^{m}\right), \nabla e_{u}\right)\right| \leq\left\|e_{u}\right\|^{2}
$$

The third and fourth terms of equation (37) are bounded by

$$
\begin{gathered}
|I I I| \leq\left|\varepsilon_{c}\left(p \partial_{x}\left(u-\bar{w}^{m}\right), e_{u}\right)\right| \leq 2 \varepsilon_{c} \varepsilon\left\|u-\bar{w}^{m}\right\|^{2}+\frac{\varepsilon_{c}}{2 \varepsilon}\left|e_{u}\right|^{2} \\
|I V| \leq q_{0}\left|u-\bar{w}^{m}\right|^{2} \leq q_{0}\left|e_{u}\right|^{2}
\end{gathered}
$$

In view of (H1) and Younge's inequality we can estimate the right hand by

$$
|V| \leq \frac{\kappa}{2}\left|f-\bar{f}^{m}\right|^{2}+\frac{1}{2 \kappa}\left|e_{u}\right|^{2} \leq \frac{\kappa}{2} \tau^{2}+\frac{1}{2 \kappa}\left|e_{u}\right|^{2}
$$

Collecting all the previous bounds, choosing $\xi, \varepsilon$, and $\kappa$ sufficiently small and applying Gronwall lemma we obtain (28). 


\section{A Full discretization scheme}

In this section, we use the exponential spline for the solution of discretization of problem (1-3) which is written in the form (denoting $y(x) \equiv w_{j}(x)$ ):

$$
-\varepsilon_{d} y^{\prime \prime}+\varepsilon_{c} p(x) y^{\prime}+(q(x)+1 / \tau) y=f_{i}+w_{j-1} / \tau, \quad x \in \Omega,
$$

with boundary conditions

$$
y=0, x \in \partial \Omega,
$$

in the time step $t_{j}, \quad j=1,2, \cdots, n, w_{j-1}$ is known and we have to solve the two parameter singularly perturbed boundary value problem

$$
-\varepsilon_{d} y^{\prime \prime}+\varepsilon_{c} p(x) y^{\prime}+G(x) y=H(x), \quad x \in \Omega,
$$

associated with the boundary condition (43). Here we use $G(x)$ and $H(x)$ to denote $(q(x)+1 / \tau)$ and $\left(f_{j}+w_{j-1} / \tau\right)$ respectively. To this end we consider a uniform mesh $\Delta$ with nodal point $x_{i}$ on $[a, b]$ such that $\Delta: a=x_{1}<x_{2}<$ $\ldots<x_{m-1}<x_{m}=b$ where

$$
x_{i}=a+i h, h=\frac{b-a}{m} i=0,1,2, \cdots, m .
$$

Let $y(x)$ be the exact solution of the problem (44)-(43) and $S_{i}$ be an approximation to $y_{i}=y\left(x_{i}\right)$ obtained by the segment $Q_{i}(x)$ passing through the points $\left(x_{i}, S_{i}\right)$ and $\left(x_{i+1}, S_{i+1}\right)$. Each mixed spline segment $Q_{i}(x)$ has the form, (for more details see [15-18]):

$$
Q_{i}(x)=a_{i} e^{k\left(x-x_{i}\right)}+b_{i} e^{-k\left(x-x_{i}\right)}+c_{i}\left(x-x_{i}\right)+d_{i}, \quad i=0,1,2, \ldots, m,
$$

where $a_{i}, b_{i}, c_{i}$ and $d_{i}$ are constants and $k$ is a free parameter.

Following [18], we get the following relation for $i=1,2, \cdots, m-1$,

$$
\left(S_{i+1}-2 S_{i}+S_{i-1}\right)=h^{2}\left(\alpha M_{i+1}+\beta M_{i}+\alpha M_{i-1}\right),
$$

where:

$\theta=k h, \alpha=(\sinh (\theta)-\theta) / \theta^{2} \sinh (\theta)$ and $\beta=(2 \theta \cosh (\theta)-2 \sinh (\theta)) / \theta^{2} \sinh (\theta)$, when $k \rightarrow 0$ that $\theta \rightarrow 0$ then $(\alpha, \beta)=\frac{1}{6}(1,4)$ and the relation defined by $(47)$ reduces to an ordinary cubic spline relation:

$$
\left(S_{i+1}-2 S_{i}+S_{i-1}\right)=\frac{h^{2}}{6}\left(M_{i+1}+4 M_{i}+M_{i-1}\right),
$$

at the point $x_{i}$ the proposed singularly perturbed problem may discretized by:

$$
M_{i}=\frac{1}{\varepsilon_{d}}\left(\varepsilon_{c} p_{i} S_{i}^{(1)}+G_{i} S_{i}+H_{i}\right),
$$

where, $S_{i}^{(1)}=\frac{S_{i+1}-S_{i-1}}{2 h}, S_{i+1}^{(1)}=\frac{3 S_{i+1}-4 S_{i}+S_{i-1}}{2 h}, S_{i-1}^{(1)}=\frac{-S_{i+1}+4 S_{i}-3 S_{i-1}}{2 h}, G_{i}=$ $G\left(x_{i}\right), H_{i}=H\left(x_{i}\right)$ and $p_{i}=p\left(x_{i}\right)$. 
Substituting (49) into (47), we get the following linear equation for every $i=$ $1,2, \cdots, m-1$

$$
\begin{aligned}
\varepsilon_{c}\left(S_{i-1}-2 S_{i}+S_{i+1}\right)+\frac{\varepsilon_{d} h}{2}\left(D_{i} S_{i-1}+E_{i} S_{i}+A_{i} S_{i+1}\right) \\
-h^{2}\left(\alpha G_{i-1} S_{i-1}+\beta G_{i} S_{i}+\alpha G_{i+1} S_{i+1}\right)=-h^{2}\left(\alpha H_{i-1}+\beta H_{i}+\alpha H_{i+1}\right) .
\end{aligned}
$$

Equation (50) gives $n-1$ linear algebraic equations in $n-1$ unknowns $S_{i}$, where $A_{i}=\alpha p_{i-1}-\beta p_{i}-3 \alpha p_{i+1}, D_{i}=3 \alpha p_{i-1}+\beta p_{i}-\alpha p_{i+1}, E_{i}=4 \alpha\left(p_{i+1}-p_{i-1}\right)$.

Now we investigate the convergence analysis of the suggested algorithm. The exponential spline solution of (1)-(3) is based on the linear equation given by (50) Let $Y=\left(y_{i}\right), S=\left(s_{i}\right), C=\left(c_{i}\right), T=\left(t_{i}\right)$ and $E=\left(e_{i}\right)=Y-S$ be $m-1$ dimensional column vectors. It is easily seen that the system of equation (50) gives $n-1$ linear algebraic equations in the $n-1$ unknowns $S_{i}, \quad i=$ $1,2, \cdots, m-1$. Then we can rewrite equation (50) in the standard matrix equation as:

$$
B Y=C+T
$$

Now the matrix $B$ may be written as

$$
B=\varepsilon_{d} B_{0}+h^{2} B_{1} F+\frac{\varepsilon_{c} h}{2} B_{2}, \text { and } F=\operatorname{diag} G_{i}
$$

where

$$
\begin{gathered}
B_{0}=\left(\begin{array}{ccccc}
2 & -1 & & & \\
-1 & 2 & -1 & & \\
& & \ddots & & \\
& & -1 & 2 & -1 \\
& -1 & 2
\end{array}\right), B_{1}=\left(\begin{array}{ccccc}
\beta & \alpha & & & \\
\alpha & \beta & \alpha & & \\
& & \ddots & & \\
& & \alpha & \beta & \alpha \\
& & & \alpha & \beta
\end{array}\right), \\
B_{2}=\left(\begin{array}{cccccc}
E_{1} & A_{1} & & & & \\
D_{2} & E_{2} & A_{2} & & \\
& & \ddots & & \\
& & D_{n-2} & E_{n-2} & A_{n-2} \\
& & & D_{n-1} & E_{n-1}
\end{array}\right)
\end{gathered}
$$

and $C_{i}= \begin{cases}-h^{2}\left(\alpha H_{0}+\beta H_{1}+\alpha H_{2}\right), & i=1 \\ -h^{2}\left(\alpha H_{i-1}+\beta H_{i}+\alpha H_{i+1}\right), & i=2,3, \ldots, n-2 \\ -h^{2}\left(\alpha H_{n-2}+\beta H_{n-1}+\alpha H_{n}\right), & i=n-1\end{cases}$

We have the local truncation error are

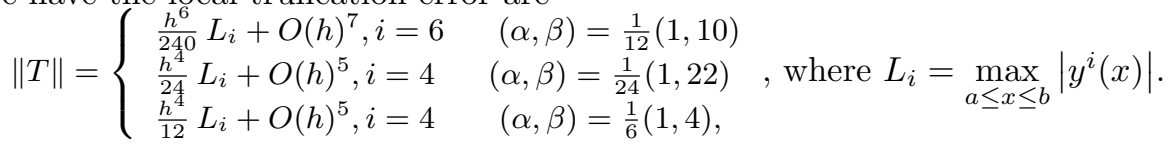


From Eq. (52), then the row sum of the matrix $B$ satisfies: for $\left|p_{i}\right| \leq P \geq 0$,

$$
\begin{aligned}
B & =\varepsilon_{d} B_{0}+\frac{\varepsilon_{c} h}{2} B_{1}+h^{2} B_{2} F \\
B_{1} & =\varepsilon_{d}+\frac{\varepsilon_{c} h}{2}(2 \alpha+\beta) P+h^{2}(2 \alpha+\beta) F i=1, n-1, \\
B_{i} & =h^{2}(2 \alpha+\beta) F, \quad i=2,3, \cdots, n-2,
\end{aligned}
$$

for small values of $\varepsilon_{d}$ and $\varepsilon_{c}$ then the matrix $B$ is irreducible and monotone and it follows that $B^{-1}$ exist thus the problem (51) has a unique solution.

Lemma $5.1([5])$. For any $0<d<1$, we have up to a certain order $q$ that it depends on the smoothness of the data

$$
\left|y^{k}(x)\right| \leq C\left\{1+\psi_{1}^{k} e^{-d \psi_{1} x}+\psi_{2}^{k} e^{-d \psi_{2}(1-x)}\right\}, \text { for } 0 \leq k \leq q
$$

\section{Shishkin Mesh Strategy}

We form the piecewise-uniform grid in such a way that more points are generated in the boundary layer regions than outside of it. We divide the interval $\bar{\Omega}=[0,1]$ into three subintervals:

$$
\Lambda_{0}=\left[0, \gamma_{1}\right], \Lambda_{c}=\left[\gamma_{1}, 1-\gamma_{2}\right] \text { and } \Lambda_{1}=\left[1-\gamma_{2}, 1\right],
$$

where transition parameters are given by

$$
\gamma_{1}=\min \left(\frac{1}{4}, \frac{2}{\psi_{1}} \ln n\right), \gamma_{2}=\min \left(\frac{1}{4}, \frac{2}{\psi_{2}} \ln n\right),
$$

where

$$
\psi_{1}=-\max _{x \in[0,1]} \eta_{1}(x) \text { and } \psi_{2}=\min _{x \in[0,1]} \eta_{2}(x), \quad \eta_{1}(x)<0 \text { and } \eta_{2}(x)>0 .
$$

the real solutions of characteristic equation

$$
-\varepsilon_{d} \eta(x)^{2}+\varepsilon_{c} p(x) \eta(x)+G(x)=0 .
$$

with $n$ to be the number of subdivision points of the interval $[0,1]$ and we place $n / 4, n / 2$ and $n / 4$ mesh points, respectively, in $\left[0, \gamma_{1}\right],\left[\gamma_{1}, 1-\gamma_{2}\right]$ and $\left[1-\gamma_{2}, 1\right]$. Denote the step sizes in each subinterval by $h_{1}=\frac{4 \gamma_{1}}{n}, h_{2}=\frac{2\left(1-\gamma_{1}-\gamma_{2}\right)}{n}$ and $h_{3}=\frac{4 \gamma_{2}}{n}$, respectively.

Where the resulting piecewise-uniform Shishkin mesh may be represented by:

$$
\tilde{h}= \begin{cases}h_{1}=h_{i}=\frac{4 \gamma_{11}}{n} & \text { if } i=1,2, \ldots \ldots, n / 4, \\ h_{2}=h_{i}=\frac{2\left(1-\gamma_{1}-\gamma_{2}\right)}{n} & \text { if } i=n / 4+1, \ldots \ldots \ldots, 3 n / 4, \\ h_{3}=h_{i}=\frac{4 \gamma_{2}}{n} & \text { if } i=3 n / 4+1, \ldots \ldots, n,\end{cases}
$$

where $n$ is the number of discretization points and set of mesh points $\bar{\Omega}_{n}=$ $\left\{x_{i}\right\}_{i=0}^{n}$ with:

$$
x_{i}= \begin{cases}x_{i-1}+h_{1} & \text { if } i=1,2, \ldots \ldots, n / 4, \\ x_{i-1}+h_{2} & \text { if } i=n / 4+1, \ldots \ldots, 3 n / 4 . \\ x_{i-1}+h_{3} & \text { if } i=3 n / 4+1, \ldots \ldots, n,\end{cases}
$$

Thus, a uniform mesh is placed on each of these subintervals. 
Lemma $5.2([5])$. The solution $y(x)$ of (42)-(43) has the representation

$$
y(x)=U(x)+E_{0}(x)+E_{1}(x) .
$$

Theorem 5.1. Let $S(x)$ be the approximate solution of the solution $y(x)$ of (42), then

$$
\|S(x)-y(x)\|_{\infty} \leq C n^{-2}(\ln n)^{2},
$$

where Cis a constant independent of $\varepsilon_{d}$ and $\varepsilon_{c}$.

Proof. The estimate is obtained on each subinterval $\Omega_{i}=[0,1]$ separately. Let any function $z$ on $\Omega_{i}$, see [5 ]

$$
\bar{z}(x)=z_{i-1}(x) \phi_{i-1}(x)+z_{i}(x) \phi_{i}(x),
$$

and so it is obvious that, on $\Omega_{i}$

$$
\bar{z}(x) \leq \max _{\Omega_{i}} z(x)\left[\phi_{i-1}(x)+\phi_{i}(x)\right]
$$

Taking maximum norm on both sides, we get

$$
|\bar{z}(x)| \leq \max _{\Omega_{i}}|z(x)|,
$$

and by appropriate Taylor expansions it is easy to see that

$$
|\bar{z}(x)-z(x)| \leq C h_{i}^{2} \max _{\Omega_{i}}\left|z^{\prime \prime}(x)\right| .
$$

From (58) and Lemma 5.1, on $\Omega_{i}$,

$$
\begin{aligned}
& |S(x)-y(x)| \leq C h_{i}^{2} \max _{\Omega_{i}}\left|y^{\prime \prime}(x)\right| \leq C h_{i}^{2}\left\{1+\psi_{1}^{2} e^{-j \psi_{1} x}+\psi_{2}^{2} e^{-j \psi_{2}(1-x)}\right\} \\
& \leq C h_{i}^{2}\left\{1+\psi_{1}^{2}+\psi_{2}^{2}\right\}
\end{aligned}
$$

Also, using Lemma 5.3 and equation (58) on $\Omega_{i}$,

$$
\begin{aligned}
|S(x)-y(x)| & \leq\left|\bar{U}+\bar{E}_{0}+\bar{E}_{1}-U-E_{0}-E_{1}\right| \\
& \leq|\bar{U}-U|+\left|\bar{E}_{0}-E_{0}\right|+\left|\bar{E}_{1}-E_{1}\right| \\
& \leq C h_{i}^{2} \max _{\Omega_{i}}\left|U^{\prime \prime}(x)\right|+2 \max _{\Omega_{i}}\left|E_{0}(x)\right|+2 \max _{\Omega_{i}}\left|E_{1}(x)\right| \\
& \leq C\left(h_{i}^{2}+e^{-j \psi_{1} x_{i}}+e^{-j \psi_{2}\left(1-x_{i}\right)}\right)
\end{aligned}
$$

Case 1. The argument now depends on whether $\frac{2 \ln n}{\psi_{1}} \leq \frac{1}{4}$ and $\frac{2 \ln n}{\psi_{2}} \leq \frac{1}{4}$. In this case $\psi_{1} \leq C \ln n$ and $\psi_{2} \leq C \ln n$. Then the result follows at once from (58)

$$
|S(x)-y(x)| \leq C n^{-2}(\ln n)^{2}
$$

Case 2. When $\gamma_{1}=\frac{2}{\psi_{1}} \ln n$ and $\gamma_{2}=\frac{2}{\psi_{2}} \ln n$. Suppose that $i$ satisfies $1 \leq$ $i \leq \frac{n}{4}$ and $\frac{3 n}{4} \leq i \leq n$. Then $h_{i}=\frac{4 \gamma_{1}}{n}=\frac{C n^{-1} \ln n}{\psi_{1}}$ and $h_{i}=\frac{4 \gamma_{2}}{n}=\frac{C n^{-1} \ln n}{\psi_{2}}$ respectively. Now from (58)

$$
|S(x)-y(x)| \leq C n^{-2}(\ln n)^{2}
$$


If $i$ satisfied $\frac{n}{4} \leq i \leq \frac{3 n}{4}$. Then $\gamma_{1} \leq x_{i}$ and $x_{i} \leq 1-\gamma_{2}$ or $\gamma_{2} \leq 1-x_{i}$ and so

$$
\begin{aligned}
& e^{-\psi_{1} x_{i}} \leq e^{-\psi_{1} \gamma_{1}}=e^{-2 \ln n}=n^{-2}, \text { since } \gamma_{1}=\frac{2 \ln n}{\psi_{1}} \\
& e^{-\psi_{2}\left(1-x_{i}\right)} \leq e^{-\psi_{1} \gamma_{2}}=e^{-2 \ln n}=n^{-2}, \text { since } \gamma_{2}=\frac{2 \ln n}{\psi_{2}}
\end{aligned}
$$

Using the above values in (59), we get the required result.

We are now in a position to formulate the main contribution of this work by combining (28) and (59) in the following theorem.

Theorem 5.2. Let the assumptions (H1)-(H2) be satisfied. Then $u^{\alpha} \rightarrow u$ in $u \in H^{1}\left(I ; L_{2}(\Omega)\right) \cap L_{2}(I ; V)$ for $\alpha \rightarrow 0$ where $u$ is the variational solution of problem (1)-(3) and $u^{\alpha}$ is constructed from the solution of problem (42)-(43). Moreover, we have the error estimates

$$
\left\|u-u^{\alpha}\right\|_{L_{2}\left(I ; L_{2}(\Omega)\right)} \leq C\left(h^{2}+\tau\right) .
$$

\section{Numerical Results}

We present a case study to illustrate the performance of the error estimation procedures of section 5. For purposes of comparison, contrast and performance, two cases with known solutions were chosen.

Case study 1: Consider the following singularly perturbed parabolic partial differential equation, see [1].

$u_{t}-\varepsilon_{d} u_{x x}+u_{x}=f(x, t), T=1$,in the interval $[0,1]$.

associated with the Dirichlet boundary conditions $u(0, t)=0, u(1, t)=0$. and the initial condition $u_{0}(x)=e^{-1 / \varepsilon}+\left(1-e^{-1 / \varepsilon}\right) x-e^{-(1-x) / \varepsilon}$, where $f(x, t)=$ $e^{-t}\left(-c_{1}+c_{2}(1-x)+e^{-(1-x) / \varepsilon}\right)$. The exact solution is given by $u(x, t)=$ $e^{-t}\left(c_{1}+c_{2} x-e^{-(1-x) / \varepsilon}\right)$, where $c_{1}=e^{-1 / \varepsilon}, c_{2}=1-e^{-1 / \varepsilon}$.

Case study 2: Consider the following singularly perturbed parabolic partial differential equation.

$u_{t}-\varepsilon_{d} u_{x x}+\varepsilon_{c} u_{x}=f(x, t), T=1$, in the interval $[0,1]$.

associated with the Dirichlet boundary conditions $u(0, t)=0, u(1, t)=0$. and the initial condition

$$
u_{0}(x)=\left[\rho_{1} \cos (\pi x)+\rho_{2} \sin (\pi x)+\psi_{1} \exp \left(\lambda_{1} x\right)+\psi_{2} \exp \left(-\lambda_{2}(1-x)\right)\right],
$$

where

$$
\begin{aligned}
f(x, t)= & e^{-t}\left[\left\{-\rho_{1} \cos (\pi x)-\rho_{2} \sin (\pi x)-\psi_{1} \exp \left(\lambda_{1} x\right)-\psi_{2} \exp \left(-\lambda_{2}(1-x)\right)\right\}\right. \\
& +\varepsilon_{d}\left\{\rho_{1} \pi^{2} \cos (\pi x)+\rho_{2} \pi^{2} \sin (\pi x)-\frac{\psi_{1}}{\lambda_{1}^{2}} \exp \left(\lambda_{1} x\right)-\frac{\psi_{2}}{\lambda_{2}^{2}} \exp \left(-\lambda_{2}(1-x)\right)\right\} \\
& \left.+\varepsilon_{c}\left\{-\rho_{1} \pi \sin (\pi x)+\rho_{2} \pi \cos (\pi x)+\frac{\psi_{1}}{\lambda_{1}} \exp \left(\lambda_{1} x\right)+\frac{\psi_{2}}{\lambda_{2}} \exp \left(-\lambda_{2}(1-x)\right)\right\}\right] .
\end{aligned}
$$


TABle 1. Maximum pointwise errors for Case study1

\begin{tabular}{|c|c|c|c|c|c|c|}
\hline $\begin{array}{c}n \\
\varepsilon_{d}\end{array}$ & 16 & 32 & 64 & 128 & 256 & 512 \\
\hline $2^{-6}$ & $2.9748-2$ & $1.4647-2$ & $8.4430-3$ & $9.1708-3$ & $9.3305-3$ & $9.1833-3$ \\
\hline $2^{-8}$ & $3.5638-2$ & $1.3000-2$ & $9.3378-3$ & $8.4218-3$ & $8.3579-3$ & $8.7259-3$ \\
\hline $2^{-10}$ & $5.1972-2$ & $1.4234-2$ & $8.3305-3$ & $7.9579-3$ & $8.1124-3$ & $8.0812-3$ \\
\hline $2^{-12}$ & $6.7088-2$ & $2.3185-2$ & $8.2129-3$ & $7.6243-3$ & $7.9618-3$ & $8.0662-3$ \\
\hline $2^{-24}$ & $7.3818-2$ & $3.4126-2$ & $1.5756-2$ & $9.1052-3$ & $8.4470-3$ & $8.3314-3$ \\
\hline $2^{-26}$ & $7.3819-2$ & $3.4128-2$ & $1.5761-2$ & $9.1078-3$ & $8.4491-3$ & $8.3337-3$ \\
\hline $2^{-28}$ & $7.3819-2$ & $3.4129-2$ & $1.5763-2$ & $9.1084-3$ & $8.4496-3$ & $8.3343-3$ \\
\hline $2^{-30}$ & $7.3819-2$ & $3.4129-2$ & $1.5763-2$ & $9.1086-3$ & $8.4497-3$ & $8.3344-3$ \\
\hline $2^{-32}$ & $7.3819-2$ & $3.4129-2$ & $1.5763-2$ & $9.1086-3$ & $8.4498-3$ & $8.3345-3$ \\
\hline
\end{tabular}

TABLE 2. Maximum pointwise errors and order of convergence for Case study1

\begin{tabular}{|c|c|c|c|c|}
\hline $\begin{array}{c}n \\
\varepsilon_{d}\end{array}$ & 16 & 32 & 64 & 128 \\
\hline $2^{-10}$ & $5.1972-2$ & $1.4234-2$ & $8.3305-3$ & $7.9579-3$ \\
\hline & $\mathbf{1 . 8 6 8 4}$ & $\mathbf{0 . 7 7 2 9}$ & $\mathbf{0 . 0 6 6 0}$ & \\
\hline $2^{-12}$ & $6.7088-2$ & $2.3185-2$ & $8.2129-3$ & $7.6243-3$ \\
\hline & $\mathbf{1 . 5 3 2 8}$ & $\mathbf{1 . 4 9 7 2}$ & $\mathbf{0 . 1 0 7 3}$ & \\
\hline $2^{-24}$ & $7.3818-2$ & $3.4126-2$ & $1.5756-2$ & $9.1052-3$ \\
\hline & $\mathbf{1 . 1 1 3 1}$ & $\mathbf{1 . 1 1 5 0}$ & $\mathbf{0 . 7 9 1 1}$ & \\
\hline $2^{-26}$ & $7.3819-2$ & $3.4128-2$ & $1.5761-2$ & $9.1078-3$ \\
\hline & $\mathbf{1 . 1 1 3 0}$ & $\mathbf{1 . 1 1 4 6}$ & $\mathbf{0 . 7 9 1 2}$ & \\
\hline $2^{-28}$ & $7.3819-2$ & $3.4129-2$ & $1.5763-2$ & $9.1084-3$ \\
\hline & $\mathbf{1 . 1 1 3 0}$ & $\mathbf{1 . 1 1 4 4}$ & $\mathbf{0 . 7 9 1 3}$ & \\
\hline $2^{-30}$ & $7.3819-2$ & $3.4129-2$ & $1.5763-2$ & $9.1086-3$ \\
\hline & $\mathbf{1 . 1 1 3 0}$ & $\mathbf{1 . 1 1 4 4}$ & $\mathbf{0 . 7 9 1 2}$ & \\
\hline $2^{-32}$ & $7.3819-2$ & $3.4129-2$ & $1.5763-2$ & $9.1086-3$ \\
\hline & $\mathbf{1 . 1 1 3 0}$ & $\mathbf{1 . 1 1 4 4}$ & $\mathbf{0 . 7 9 1 2}$ & \\
\hline
\end{tabular}

The exact solution is given by

$$
u(x, t)=e^{-t}\left[\rho_{1} \cos (\pi x)+\rho_{2} \sin (\pi x)+\psi_{1} \exp \left(\lambda_{1} x\right)+\psi_{2} \exp \left(-\lambda_{2}(1-x)\right)\right],
$$

where $\rho_{1}=\frac{\varepsilon_{d} \pi^{2}+1}{\varepsilon_{c}^{2} \pi^{2}+\left(\varepsilon_{d} \pi^{2}+1\right)^{2}}, \rho_{2}=\frac{\varepsilon_{c} \pi}{\varepsilon_{c}^{2} \pi^{2}+\left(\varepsilon_{d} \pi^{2}+1\right)^{2}}, \psi_{1}=-\rho_{1} \frac{1+\exp \left(-\lambda_{2}\right)}{1-\exp \left(\lambda_{1}-\lambda_{2}\right)}, \psi_{2}=$ $\rho_{1} \frac{1+\exp \left(\lambda_{1}\right)}{1-\exp \left(\lambda_{1}-\lambda_{2}\right)}, \lambda_{1}=\frac{\varepsilon_{c}-\sqrt{\varepsilon_{c}^{2}+4 \varepsilon_{d}}}{2 \varepsilon_{d}}, \lambda_{2}=\frac{\varepsilon_{c}+\sqrt{\varepsilon_{c}^{2}+4 \varepsilon_{d}}}{2 \varepsilon_{d}}$.

All the computations were performed by using MATLAB 7 . 
TABle 3. Comparison of maximum pointwise errors for Case study1

\begin{tabular}{|c|c|c|c|c|c|c|c|c|}
\hline \multirow{2}{*}{$\varepsilon_{d}$} & \multicolumn{2}{|l|}{32} & \multicolumn{2}{|l|}{64} & \multicolumn{2}{|l|}{128} & \multicolumn{2}{|l|}{256} \\
\hline & $\begin{array}{l}\text { Proposed } \\
\text { scheme }\end{array}$ & $\begin{array}{l}\text { Scheme } \\
\text { in [1] }\end{array}$ & $\begin{array}{l}\text { Proposed } \\
\text { scheme }\end{array}$ & $\begin{array}{l}\text { Scheme } \\
\text { in [1] }\end{array}$ & $\begin{array}{l}\text { Proposed } \\
\text { scheme }\end{array}$ & $\begin{array}{l}\text { Scheme } \\
\text { in [1] }\end{array}$ & $\begin{array}{l}\text { Proposed } \\
\text { scheme }\end{array}$ & $\begin{array}{l}\text { Scheme } \\
\text { in [1] }\end{array}$ \\
\hline $2^{-8}$ & $1.4647-2$ & $4.5409-2$ & $8.4430-3$ & $2.7112-2$ & $9.1708-3$ & $1.5275-2$ & $9.3305-3$ & $8.3396-3$ \\
\hline $2^{-10}$ & $1.3000-2$ & $4.8331-2$ & $9.3378-3$ & $2.9637-2$ & $8.4218-3$ & $1.7085-2$ & $8.3579-3$ & $9.4669-3$ \\
\hline $2^{-12}$ & $1.4234-2$ & $4.9125-2$ & $8.3305-3$ & $3.0386-2$ & $7.9579-3$ & $1.7720-2$ & $8.1124-3$ & $9.4669-3$ \\
\hline $2^{-24}$ & $2.3185-2$ & $4.9395-2$ & $8.2129-3$ & $3.0647-2$ & $7.6243-3$ & $1.7952-2$ & $7.9618-3$ & $1.0184-2$ \\
\hline $2^{-26}$ & $3.4126-2$ & $4.9395-2$ & $1.5756-2$ & $3.0647-2$ & $9.1052-3$ & $1.7952-2$ & $8.4470-3$ & $1.0184-2$ \\
\hline
\end{tabular}

TABle 4. Maximum errors for Case study2 $n=128$ and $\tau=$ $1.6 /(n+1)^{2}$

\begin{tabular}{|c|c|c|c|}
\hline$\varepsilon_{c}$ & $\varepsilon_{d}=10^{-2}$ & $\varepsilon_{d}=10^{-4}$ & $\varepsilon_{d}=10^{-6}$ \\
\hline $10^{-3}$ & $3.2784-2$ & $2.9841-2$ & $2.8807-2$ \\
\hline $10^{-4}$ & $4.5702-2$ & $4.7121-2$ & $4.6675-2$ \\
\hline $10^{-5}$ & $5.3734-2$ & $6.6223-2$ & $6.8517-2$ \\
\hline
\end{tabular}

To compute the experimental rates of convergence $O r d^{n}$ for every fixed $\varepsilon$, we use the rate of convergence from:

$$
O r d^{n}=\frac{\ln E^{n}-\ln E^{2 n}}{\ln 2}
$$

The numerical results displayed in Tables 1-3 clearly indicate that the proposed method based on an exponential-spline function with Shishkin mesh is $\varepsilon$-uniformly convergent. Numerical solution profiles for case study are given in figure 1 , respectively, for $n=128, \tau=0.02$ and different values of $\varepsilon_{d}=1,2^{-6}$ and $2^{-12}$. For comparison, our results are better than the numerical solution presented in Clavero et al. [1]. Numerical solution profiles for case study2 are given in table 4 and also figure 2 and 3 for different values of $\varepsilon_{d}$ and $\varepsilon_{c}$.

It is clearly that all solutions are in good harmony with each approximate and exact solution and the convergence obtained is parameter uniform and satisfies the theoretical predictions.

\section{Conclusion}

In this paper, we have investigated the application of Rothe's approximation in time discretization and the exponential spline approximation in the spatial discretization to solve the convection-diffusion problems. The method is shown to be uniformly convergent i.e., independent of mesh parameters and perturbation parameters $\varepsilon_{d}$ and $\varepsilon_{c}$. It has also been seen that the accuracy in the numerical results for the proposed scheme is comparable to that obtained by the uniformly convergent method with a uniform mesh [1]. It has been found that the proposed algorithm gives highly accurate numerical results and higher order of convergence than an upwind finite difference scheme. The present method 
provides a very simple and accurate method for solving convection-diffusion problems.
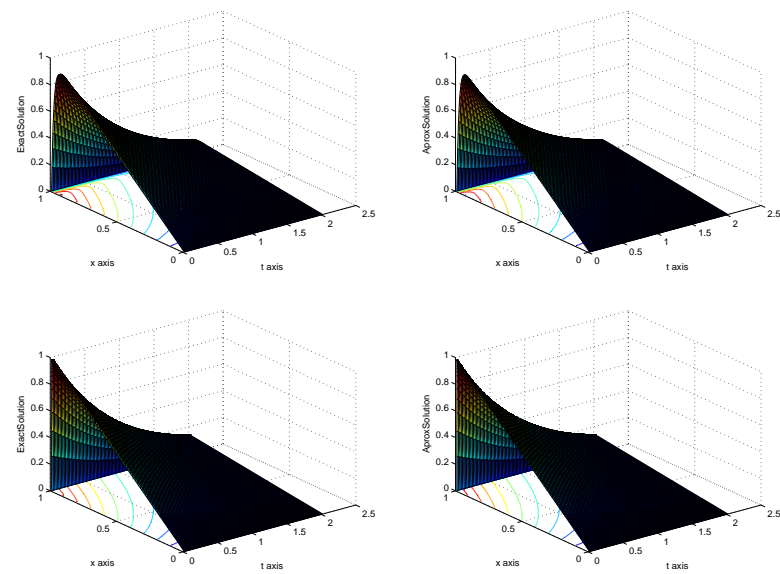

FIGURE 1. Exact and approximate solutions profiles of the case study1 for $n=128, \tau=0.02$ and $\varepsilon_{d}=2^{-6}$ and $\varepsilon_{d}=2^{-12}$ respectively
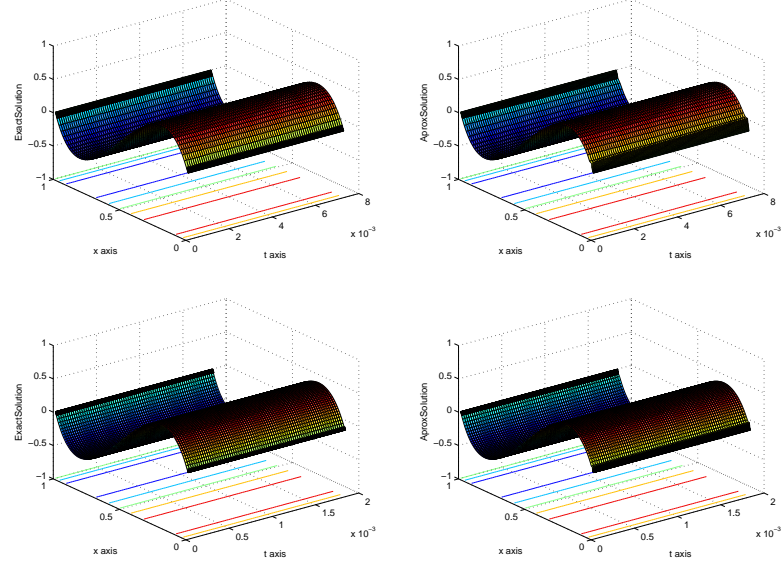

FiguRE 2. Exact and approximate solutions profiles of the case study2 for $n=128,256, \tau=1.6 /(n+1)^{2}, \varepsilon_{c}=2^{-6}$, and $\varepsilon_{d}=$ $2^{-6}$. 

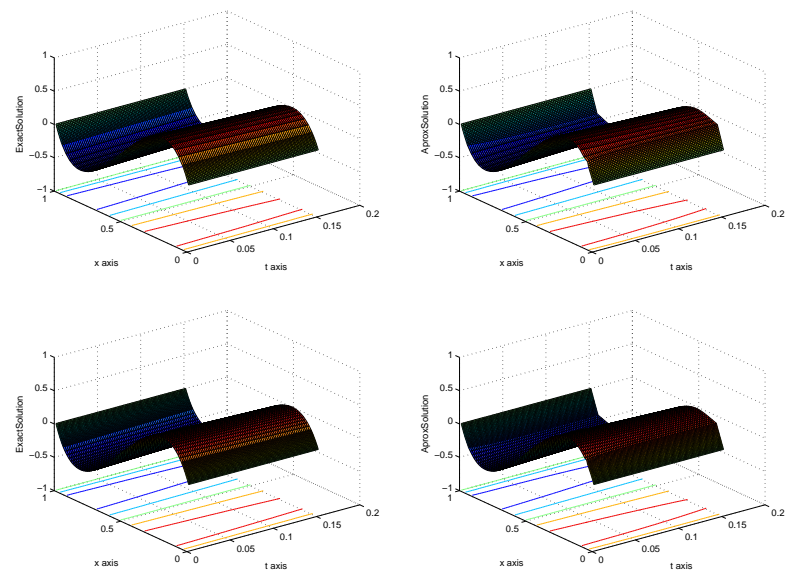

FIGURE 3. Exact and approximate solutions profiles of the case study2 for $n=64,128, \tau=0.002, \varepsilon_{c}=2^{-6}$, and $\varepsilon_{d}=2^{-6}$.

\section{REFERENCES}

1. C. Clavero, J.C. Jorge, F. Lisbona, Uniformly convergent scheme on a nonuniform mesh for convection-diffusion parabolic problems, J. Comput. Appl. Math. 154, 415-429, 2003.

2. N.G. Amin, M.S. El-Azab, A new linear scheme for solving the 1 D Burger's equation, American Academic and Scholarly Research Journal Vol. 4, No. 2, March 2012.

3. W. Jager, J. Kacur, "Approximation of porous medium type systems by nondegenerateelliptic systems" Preprint No. 582,Universitat Heidelberg, 1990.

4. M.K. Kadalbajoo, V. Gupta, and A. Awasthi. A uniformly convergent B-Spline collocation method on a nonuniform mesh for singularly perturbed one-dimensional time-dependent linear convection-diffusion problem, Journal of Computational and Applied Mathematics, $220,271-289,2008$.

5. M.K. Kadalbajoo, A.S Yadaw, B-Spline collocation method for a two-parameter singularly perturbed convection-diffusion boundary value problem. Applied Mathematics and Computation, 201, 504-513, 2008.

6. A. Kufner, O. John, S.Fucik, "Function Spaces" Nordhoff, Leyden, 1997.

7. J.I. Ramos, An exponentially fitted method for singularly perturbed, one-dimensional, parabolic problems, Appl. Math. Comput. 161, 513-523, 2005.

8. J.Rashidinia, M.Ghasemi, and R.Jalilian, A collocation method for the solution of nonlinearone-dimensional parabolic equations, Mathematical Sciences,4, 1, 87-104, 2010.

9. S.C.S Rao, and M. Kumar, Exponential B-spline collocation method for self -adjoint singularly perturbed boundary value problems, Applied Numerical Mathematics, 58, 1572-1581, 2008.

10. S.C.S Rao, and M. Kumar, Parameter uniformly exponential spline difference scheme for singularly perturbed semilinear reaction diffusion problems, Nonlinear Analysis, 71, 15791588,2009

11. M. Sakai, R.A. Usmani, On exponential splines, J. approx. theory, 47, 122-131, 1986.

12. M. Sakai, R.A. Usmani, A class of simple exponential B-splines and their applications to numerical solution to singular perturbation problems, Numer. Math., 55, 493-500, 1989. 
13. K. Surla, V. Jerkovic, Some possibilities of applying spline collocations to singular perturbation problems, Numerical Methods and Approximation Theory, vol. II, Novisad, pp. 19-25, 1985.

14. M. Sharma and A. Kaushik, Convergence Analysis of Weighted Difference Approximations on Piecewise Uniform Grids to a Class of Singularly Perturbed Functional Differential Equations, J Optim Theory Appl, 155, 252-272, 2012.

15. W.K. Zahra, Exponential spline solutions for a class of two point boundary value problems over a semi-infinite range, Numer. Algor. 52,561-573, 2009. Zahra W.K., A

16. smooth approximation based on exponential spline solutions for nonlinear fourth order two point boundary value problems, Applied Mathematics andComputation 217, 8447$8457,2011$.

17. W.K. Zahra, Finite-difference technique based on exponential splines for the solutionof obstacle problems, International Journal of Computer Mathematics, 88:14, 3046-3060, 2011.

18. W.K. Zahra and A.M. El Mhlawy, Numerical Solution of Two-Parameter Singularly Perturbed Boundary Value Problems via Exponential Spline, Journal of King Saud University (Science), (2013), http://dx.doi.org/10.1016/j.jksus.2013.01.003.

W. K. Zahra received M.Sc. and Ph.D. from Faculty of Engineering, Tanta University, Tanta, Egypt. He is currently working as an Assistant Professor at Faculty of Engineering, Tanta university,Tanta, Egypt. His research interests include Numerical Methods for Singularly Perturbed Differential Equations.

Department of Physics and Engineering Mathematics, Faculty of Engineering, Tanta Univ., Tanta, Egypt.

e-mail: waheed_zahra@yahoo.com, wzahra@f-eng.tanta.edu.eg

M. S. El-Azab received M.Sc. from Faculty of Engineering, AlMansurah Univ., Al Mansurah, Egypt and Ph.D. from Comenius University, Slovakia. He is currently working as a Professor at Faculty of Engineering, AlMansurah Univ., Al Mansurah, Egypt. His research interests include Numerical Methods for Singularly Perturbed Differential Equations.

Department of Mathematics, Faculty of Engineering, AlMansurah Univ., Al Mansurah, Egypt.

Ashraf M. El Mhlawy received M.Sc. from Faculty of Engineering, Tanta University, Tanta, Egypt. His research interests include Numerical Methods for Singularly Perturbed Differential Equations.

Department of Physics and Engineering Mathematics, Faculty of Engineering, Tanta Univ., Tanta, Egypt. 